\title{
A Comparison of Outcome after Fixation of Unstable Lateral Third Clavicle Fractures, with Clavicular Hook Plate versus Precontoured Anatomical Locking Plate
}

\author{
Milan Das ${ }^{1}$, Md. Emdad Hossain², Kaushik Banerjee ${ }^{3}$, Ranjit Shaw ${ }^{4}$ \\ ${ }^{1}$ Department of Orthopaedics, Udainarayanpur S. G. Hospital, Howrah, West Bengal, India. ${ }^{2}$ Department of \\ Orthopaedics, Rampurhat Government Medical College, Rampurhat, West Bengal, India. ${ }^{3}$ Department of Orthopaedics, \\ R G Kar Medical College, Kolkata, West Bengal, India. ${ }^{4}$ Department of Orthopaedics, R G Kar Medical College, Kolkata, \\ West Bengal, India.
}

\section{ABSTRACT}

\section{BACKGROUND}

Lateral end clavicle fractures are of three types (Neer type- I, II, \& III). In Neer TypeII, the fractures are very unstable due to damage to the coracoclavicular (CC) ligament. So, it is always treated surgically. This comparative cross-sectional prospective study compares the outcome after internal fixation of these fractures with clavicular hook plate and precontoured anatomical locking plate.

\section{METHODS}

Thirty-two (32) patients attending the OPD and ER of R.G. Kar Medical College, Kolkata, having Neer Type-II fractures, were treated surgically within three weeks of injury using either clavicular hook plate (17 patients) or precontoured anatomical locking plate (15 patients) and then systematically reviewed. The relevant literature of the two fixation methods were studied to note union, infection, hardware prominence, implant failure, metaphyseal fracture and other complications. Constant-Murley score was also used for evaluation.

\section{RESULTS}

In our study, with both the fixation modalities, union was achieved in all cases by three months. No impingement was noted. Patients having fixation with precontoured anatomical locking plate had better scores and returned earlier to better function than the other group ( $\mathrm{p}$ value $<0.0001$ ).

\section{CONCLUSIONS}

Precontoured anatomical locking plate might be a better option which provides better functional outcome.

\section{KEY WORDS}

Unstable Distal Third Clavicle Fracture, Clavicular Hook Plate, Precontoured Anatomical Locking Plate
Corresponding Author: Dr. Md. Emdad Hossain, \#15, Kabitirtha Sarani, Block-Wave, Flat 9A, Kolkata-700023,

West Bengal, India.

E-mail: emdad_hssn@yahoo.in

DOI: $10.14260 /$ jemds/2020/388

Financial or Other Competing Interests: None.

How to Cite This Article:

Das M, Hossain ME, Banerjee $K$, et al. A comparison of outcome after fixation of unstable lateral third clavicle fractures, with clavicular hook plate versus precontoured anatomical locking plate. J. Evolution Med. Dent. Sci. 2020;9(23): 1771-1774, DOI: 10.14260/jemds/2020/388

Submission 25-01-2020, Peer Review 11-05-2020,

Acceptance 19-05-2020, Published 08-06-2020. 


\section{BACKGROUND}

Fractures of clavicle are not uncommon in young individuals, particularly those who take part in contact sports like football or sustain high velocity trauma during racing sports. They constitute about $2.6 \%$ of all fractures. The yearly incidence of this fracture is 29 per one lac population. ${ }^{1}$

Maximal clavicle fractures (80\% to $85 \%)$ are found in the midshaft of the bone, where the compressive forces acting on the shoulder and the narrow cross section of the bone combine to break the bone.2,3 Second most common clavicular fracture site is in the distal third region (20\%). Mechanism of injury may be the same as with midshaft fractures, but it may also happen in more elderly individuals as a result of simple falls. 4,5,6,7

The classification and management of distal clavicle fractures is dependent on the position of the fracture in relation with the conoid and the trapezoid part of the coracoclavicular (CC) ligament. According to Neer, distal clavicle fractures are of three types. Type-I: Fractures in between acromioclavicular (AC) joint and CC ligament, with CC ligament remaining intact. Type-II: Fractures associated with torn CC ligament where sometimes the trapezoid part remains intact on the distal part. Type-III: Fracture line involving the AC joint. As type-I and type-III are stable, they can be sometimes managed conservatively. On the other hand, due to unstable nature in a case of Type-II fracture, non-operative treatment frequently results in non-union. ${ }^{8}$ So, for Type-II fractures, surgery has been regarded as the treatment of choice.

Regarding implants many options have been tried as found in literature like K-wires, ${ }^{9}$ Screw fixation, ${ }^{10}$ Hook plates $^{11,12,13,14}$ and Locking plates. ${ }^{15,16,17}$ Orthopaedic surgeons are often in a dilemma while planning treatment of distal third clavicle fractures. The unstable nature of these fractures makes them prone to non-union and may hamper normal shoulder function. Although many treatment options are available in the literature, there is no gold standard protocol for treating this injury till date.

\section{METHODS}

After designing this institution based prospective comparative study, we got approval from the Institutional Ethics Committee. Then we made an "Informed Consent" format for the purpose of information, consent and counselling of the patient and his or her relatives. 32 patients of either sex, aged between 18 years to 65 years who were clinically and radiologically diagnosed to have traumatic unstable distal clavicle fractures, within three weeks from date of trauma, had been recruited for this study in R.G. Kar Medical College, Kolkata, over a period of 13 months (May, 2016 to June, 2017). We included patients with Neer TypeII ${ }^{17}$ fractures only excluding pathological fractures and persons with pre-existing shoulder pathology or medical comorbidities. Fixation method was decided according to the nature and location of the fracture. Fractures nearer to the AC joint or with comminution were treated by hook plate whereas the more medially located, non-comminuted fractures were treated with precontoured anatomical locking plate.

\section{Hook Plate Group}

After proper anaesthesia with the patient in supine position and a bolster in the interscapular region, the fracture site was opened through the anterior approach. Then open reduction was done, and the plate portion was applied over the clavicle and hook portion was introduced into the subacromial space through a tunnel made behind the AC joint. Plate bender was used when necessary.

\section{Precontoured Anatomical Locking Plate Group}

After proper anaesthesia patient position and surgical approach was the same as the hook plate group. Open reduction and provisional fixation were done with k-wires and finally the precontoured anatomical locking plate was applied placing maximum possible number of locking screws in the lateral fragment. Appropriate attention was given to avoid encroaching the $\mathrm{AC}$ joint.

\section{Postoperative Protocol}

For both the groups, the operated limb was kept in collar and cuff sling and early mobilisation exercises were started from third postoperative day. Nearly normal range of shoulder movement was noted within three to four weeks after operation. Vigorous activities were not allowed until radiological union was achieved.

\begin{abstract}
Statistics
Microsoft Excel spreadsheet was used for data entry and the analysis was done by SPSS 20.4 as well as GraphPad Prism version 5. Data had been summarised as mean and standard deviation for numerical variables and counting and percentages for categorical variables. Student's t test, chi square test and one-way analysis of variance (one-way Anova) were used. p-value $\leq 0.05$ was considered as statistically significant.
\end{abstract}

\section{RESULTS}

After satisfying the inclusion and exclusion criteria 32 patients were selected for this study. In the precontoured anatomical locking plate group there were 15 patients and in the hook plate group there were 17 patients. The mean age of locking plate group was 38.93 years and for hook plate it was 44.35 years. There were 10 female patients (hook plate 8 , precontoured anatomical locking plate 2) and 22 male patients (hook plate 9 and locking plate 13). Left sided fractures were 19 (hook plate 8 and precontoured anatomical locking plate 11) and right sided were 13 (hook plate 6; precontoured anatomical locking plate 7). The mean of the time of surgery since injury, in days was 9.0 for hook plate (minimum 4, maximum 17) and in case of precontoured anatomical locking plate was 9.8 (minimum 4, maximum 17) (table 1). In both the fixation modalities $100 \%$ union was 
achieved at 3 months (table 2).One patient had superficial infection in the precontoured anatomical locking plate group (table 3) who was treated with antibiotics, and the infection resolved uneventfully. Mean Constant Murley score in the hook plate group was 82.0 with standard deviation of 3.446 and in the precontoured anatomical locking plate group was 87.6 with standard deviation of 2.6132. (Table 4).

\begin{tabular}{|cccccccc|}
\hline $\begin{array}{c}\text { Mode of } \\
\text { Fixation } \\
\text { (Mof) }\end{array}$ & No. Mean & SD & Minimum Maximum & Median & $\begin{array}{c}\text { P- } \\
\text { Value }\end{array}$ \\
$\begin{array}{c}\text { Hook plate } \\
\text { Locking plate }\end{array}$ & 17 & 9.0000 & 3.6401 & 4.0000 & 17.0000 & 8.0000 & 0.5509 \\
\hline & 9.8000 & 3.8582 & 4.0000 & 17.0000 & 12.0000 & \\
\hline
\end{tabular}

Difference of mean time of surgery since injury in days versus mode of fixation in two groups was not statistically significant $(\mathrm{p}$-value $=0.5509$ ).

\begin{tabular}{|ccccc|}
\hline $\begin{array}{c}\text { Mode of } \\
\text { Fixation }\end{array}$ & Number & $\begin{array}{c}\text { Union at 03 } \\
\text { Months }\end{array}$ & $\begin{array}{c}\text { Non } \\
\text { Union }\end{array}$ & Percentage \\
Hook plate & 17 & 17 & 0 & $100 \%$ \\
Locking plate & 15 & 15 & 0 & $100 \%$ \\
Total & $\mathbf{3 2}$ & $\mathbf{3 2}$ & $\mathbf{0}$ & $\mathbf{1 0 0} \%$ \\
\hline Table 2. Association between Union at & Three Months \\
and Mode of Fixation \\
\hline
\end{tabular}

\begin{tabular}{|cccc|}
\hline Infection & Hook Plate & Locking Plate & Total \\
No & 17 & 14 & 31 \\
Row $\%$ & 54.8 & 45.2 & 100.0 \\
Col $\%$ & 100 & 93.3 & 96.9 \\
Yes & 0 & 1.0 & 1.0 \\
Row $\%$ & 0.0 & 100.0 & 100.0 \\
Col \% & 0.0 & 6.7 & 3.1 \\
Total & 17 & 15 & 32 \\
Row $\%$ & 53.1 & 46.9 & 100.0 \\
Col \% & 100 & 100 & 100 \\
\hline Table 3. Association between Infection and Mode of Fixation (MoF) \\
\hline Chi-square value: 1.1699; p-value: 0.2794
\end{tabular}

Difference of mean for infection versus mode of fixation in two groups was not statistically significant (p-value: 0.2794 )

\begin{tabular}{|cccccccc|}
\hline $\begin{array}{c}\text { Mode of } \\
\text { Fixation }\end{array}$ & No. & Mean & SD & Minimum & Maximum Median P-Value \\
Hook plate & 17 & 82.0000 & 3.4460 & 76.0000 & 88.0000 & 82.0000 & p-value< \\
Locking plate & 15 & 87.6000 & 2.6132 & 82.0000 & 91.0000 & 88.0000 & \\
\hline Table 4. Distribution of Mean Constant Murley Score (CMS) versus \\
Mode of Fixation (MOF) Difference of Mean CMS versus MOF in the \\
Two Groups was Statistically Significant ( $\boldsymbol{p}<0.0001)$ \\
\hline
\end{tabular}

\section{DISCUSSION}

In cases of Neer Type-II fractures, sternocleidomastoid muscle acting from above on the medial fragment and gravity pulling the distal fragment downwards along with the pull of the pectoralis major (particularly if CC ligament is deficient) results in significant displacement of the fragments. So, chances of non-union is higher if these fractures are treated non-operatively.9,18

The hook plate and precontoured anatomical locking plate are relatively new generation of implants and were used in a few recent studies. Part of the hook plate fixed to the medial fragment neutralises upward distracting forces, whereas the hook which is placed under the acromion neutralises downward forces on the distal part. So, a very high percentage of union rates were noted in most of the recent studies. Few complications were also reported like implant failure, acromial fracture, metaphyseal fracture, hook migration, subacromial impingement and rotator cuff damage. ${ }^{18,19}$ So while using this implant we must be aware and remain very careful to avoid these complications.

$74 \%$ of hook plate group had complained of mild to moderate shoulder pain in a recent study by Tan et al. ${ }^{20}$ In another study, Kashii et al reported that one patient developed acromion fracture and there was hook cut-out in 19 patients. Subacromial morphometric assessment of clavicle hook plate in cadaveric model was done by Elmaraghy et al. ${ }^{21}$

They noted that although the orientation of the hook is posterior, it remains in the middle of the supraspinatus fossa. Sometimes there may be inflammation of the subacromial bursa which further compromises the subacromial space leading to impingement of the rotator cuff. In addition to all these, majority of the authors recommended removal of the hook plate after achieving radiological union to negate the risk of osteolysis of acromion and subacromial rotator cuff impingement.

In another study, Klein et al ${ }^{22}$ noted a complication rate of $22.7 \%$ in patients treated with hook plate. Hook plates were removed after clinical and radiological confirmation of union, on an average after seven months from surgery. Interestingly, Largo et $\mathrm{al}^{23}$ reported that $53 \%$ of implant removal was the result of cultural belief and not because of cosmesis or complications.

In our study, we observed $100 \%$ union rate without rotator cuff impingement. However, we found partial loss of reduction in three patients and one patient had symptomatic hardware prominence producing a complication rate of $23.5 \%$.

With the introduction of locking plate concept, precontoured anatomical clavicular locking plates are now available for providing better fixation with lesser complications like symptomatic hardware which requires removal. ${ }^{16}$ According to Tan et $\mathrm{al}^{21}$ use of locking plate often gives excellent results in cases of Neer Type-II fractures.

In our study, precontoured anatomical locking plate group showed very good functional outcome with high union rate and with only one infected case (only $3.1 \%$ of cases; $\mathrm{p}=0.2974$ ) which resolved uneventfully during the follow up period. With our treatment strategy we achieved very high union rate $(100 \%)$ and very low complication rate. Our results were at par with those found in the recent literature without any non-union.

However, we observed statistically significant difference in Constant Murley Score between the two groups, with the locking plate group returning earlier to their pre-injury level of activity.

We thoroughly scrutinised and meticulously compared the difference in postoperative clinical and radiological findings for both the groups from the relevant literature. Observation revealed no significant difference in non-union rate. However, we found lower complication rate in locking plate group in our study.

As there were no uniform criteria to assess functional outcome in the literature, it was very difficult to analyse and compare the results in this regard. Subsequently, we used Constant Murley scoring system to compare the functional 
results and we observed statistically significant difference between the two groups.

\section{CONCLUSIONS}

Although the number of patients recruited for our study was small, we can conclude that pre-contoured anatomical locking plate and hook plate can yield very good results if used within 3 weeks from injury for Neer Type-II clavicle fractures. Locking plate can lead to earlier return to full functional outcome within 3 months after surgery with lesser complication rate when compared to the hook plate group.

\section{REFERENCES}

[1] Robinson CM. Fractures of the clavicle in the adult. Epidemiology and classification. J Bone Joint Surg $\mathrm{Br}$ 1998;80(3):476-84.

[2] Craig EV. Fractures of the clavicle. In: Rockwood CA, Matsen FA, eds. The Shoulder. $3^{\text {rd }}$ edn. Philadelphia: WB Saunders 1998: p. 428-82.

[3] Stanley D, Trowbridge EA, Norris SH. The mechanism of clavicular fracture. A clinical and biochemical analysis. J Bone Joint Surg Br 1988;70(3):461-4.

[4] Goldberg JA, Bruce WJ, Sonnabend DH, et al. Type 2 fractures of the distal clavicle: a new surgical technique. J Shoulder Elbow Surg 1997;6(4):380-2.

[5] Rockwood CA. Fractures of the outer clavicle in children and adults. J Bone Joint Surg Br 1982;64:642.

[6] Rokito AS, Eisenberg DP, Gallagher MA, et al. A comparison of non-operative and operative treatment of type II distal clavicle fractures. Bull Hosp Joint Dis 20022003;61(1-2):32-9.

[7] Webber MC, Haines JF. The treatment of lateral clavicle fractures. Injury 2000;31(3):175-9.

[8] Deafenbaugh MK, Dugdale TW, Staeheli JW, et al. Nonoperative treatment of Neer type II distal clavicle fractures: a prospective study. Contemp Orthop 1990;20(4):405-13.

[9] Kao FC, Chao EK, Chen $\mathrm{CH}$, et al. Treatment of distal clavicle fracture using Kirschner wires and tension-band wires. J Trauma 2001;51(3):522-5.
[10] Macheras G, Kateros KT, Savvidou OD, et al. Coracoclavicular screw fixation for unstable distal clavicle fractures. Orthopaedics 2005;28(7):693-6.

[11] Muramatsu K, Shigetomi M, Matsunaga T, et al. Use of the AO hook-plate for treatment of unstable fractures of the distal clavicle. Arch Orthop Trauma Surg 2007;127(3):191-4.

[12] Tambe AD, Motkur P, Qamar A, et al. Fractures of the distal third of the clavicle treated by hook plating. Int Orthop 2006;30(1):7-10.

[13] Kashii M, Inui H, Yamamoto K. Surgical treatment of distal clavicle fractures using the clavicular hook plate. Clin Orthop Relat Res 2006;447:158-64.

[14] Renger RJ, Roukema GR, Reurings JC, et al. The clavicle hook plate for Neer type II lateral clavicle fractures. J Orthop Trauma 2009;23(8):570-4.

[15] Andersen JR, Willis MP, Nelson R, et al. Pre-contoured superior locked plating of distal clavicle fractures: a new strategy. Clin Orthop Relat Res 2011;469(12):3344-50.

[16] Schliemann B, Roßlenbroich SB, Schneider KN, et al. Surgical treatment of vertically unstable lateral clavicle fractures (Neer 2b) with locked plate fixation and coracoclavicular ligament reconstruction. Arch Orthop Trauma Surg 2013;133(7):935-9.

[17] Pujalte GGA, Housner JA. Management of clavicle fractures. Curr Sports Med Rep 2008;7(5):275-80.

[18] Huang JI, Toogood P, Chen MR, et al. Clavicular anatomy and the applicability of pre-contoured plates. J Bone Joint Surg Am 2007;89(10):2260-5.

[19] Meda PVK, Machani B, Sinopidis C, et al. Clavicular hook plate for lateral end fractures: a prospective study. Injury 2006;37(3):277-83.

[20] Tan HL, Zhao JK, Qian C, et al. Clinical results of treatment using a clavicular hook plate versus a T-plate in neer type II distal clavicle fractures. Orthopedics 2012;35(8):e1191-e7.

[21] ElMaraghy AW, Devereaux MW, Ravichandiran K, et al. Subacromial morphometric assessment of the clavicle hook plate. Injury 2010;41(6):613-9.

[22] Klein SM, Badman BL, Keating CJ, et al. Results of surgical treatment for unstable distal clavicular fractures. J Shoulder Elbow Surg 2010;19(7):1049-55.

[23] Largo RD, Rudin MA, Hotz TK, et al. Anatomic reconstruction of unstable lateral clavicular fracture. J Trauma 2011;71(5):1348-51. 